\title{
Leishmania exosomes deliver preemptive strikes to create an environment permissive for early infection
}

\author{
Judith Maxwell Silverman' and Neil E. Reiner ${ }^{2,3 *}$ \\ ${ }^{1}$ Brain Research Center, Division of Neurology, Department of Medicine, University of British Columbia, Vancouver, BC, Canada \\ ${ }^{2}$ Division of Infectious Diseases, Department of Medicine, University of British Columbia, Vancouver, BC, Canada \\ ${ }^{3}$ Vancouver Coastal Heath Research Institute, University of British Columbia, Vancouver, BC, Canada
}

\section{Edited by:}

Albert Descoteaux, Institut National

de la Recherche Scientifique, Canada

\section{Reviewed by:}

Dario S. Zamboni, Universidade de

São Paulo, Brazil

Lisa A. Morici, Tulane University

School of Medicine, USA

\section{*Correspondence:}

Neil E. Reiner, Division of Infectious Diseases, Vancouver General

Hospital, Heather Pavilion

East-D452, Vancouver, BC, Canada

V5J 375.

e-mail: ethan@mail.ubc.ca
Herein, we review evidence supporting a role for Leishmania exosomes during early infection. We suggest a model in which Leishmania secreted microvesicles released into the extracellular milieu deliver effector cargo to host target cells. This cargo mediates immunosuppression and functionally primes host cells for Leishmania invasion. Leishmania ssp. release microvesicles and the amount of vesicle release and the specific protein cargo of the vesicles is sensitive to changes in environmental conditions that mimic infection. Leishmania exosomes influence the phenotype of treated immune cells. For example, wildtype (WT) exosomes attenuate interferon- $\gamma$-induced pro-inflammatory cytokine production (TNF- $\alpha$ ) by Leishmania-infected monocytes while conversely enhancing production of the anti-inflammatory cytokine IL-10. The Leishmania proteins GP63 and elongation factor-1 $\alpha$ $(E F-1 \alpha)$ are found in secreted vesicles and are likely important effectors responsible for these changes in phenotype. GP63 and EF-1 $\alpha$ access host cell cytosol and activate multiple host protein-tyrosine phosphatases (PTPs). Activation of these PTPs negatively regulates interferon- $\gamma$ signaling and this prevents effective expression of the macrophage microbicidal arsenal, including TNF- $\alpha$ and nitric oxide. In addition to changing macrophage phenotype, WT vesicles dampen the immune response of monocyte-derived dendritic cells and CD4+ $T$ lymphocytes. This capacity is lost when the protein cargo of the vesicles is modified, specifically when the amount of GP63 and EF-1 $\alpha$ in the vesicles is reduced. It appears that exosome delivery of effector proteins results in activation of host PTPs and the negative regulatory effects of the latter creates a pro-parasitic environment. The data suggest that Leishmania exosomes secreted upon initial infection are capable of delivering effector cargo to naïve target cells wherein the cargo primes host cells for infection by interfering with host cell signaling pathways.

Keywords: Leishmania, exosomes, immunosuppression, early infection, cytokine, secretion, vesicles

\section{INTRODUCTION}

The past decade has seen a significant expansion in our knowledge of Leishmania secreted proteins. In the past few years alone, seven reports addressing some aspect of the Leishmania secretome have been published (Revest et al., 2008; Silverman et al., 2008, 2010a; DebRoy et al., 2010; Kima et al., 2010; Paape et al., 2010; Hassani et al., 2011). The rationale driving this research has been that these protozoan parasites are likely dependent on secretion of effector molecules into the host extra- and intracellular environments for initiation and maintenance of successful infection. This theory is based on investigations of individual secreted proteins (VannierSantos et al., 1995; Hubel et al., 1997; Klein et al., 1999; Joshi et al., 2002; Lieke et al., 2008; Gomez et al., 2009; Halle et al., 2009; Silverman et al., 2010b; Jaramillo et al., 2011) and proteins mechanistically involved in the secretion pathway (Besteiro et al., 2006), which showed reduced virulence after loss of protein function or secretion. It has been known for some time that these eukaryotic organisms use a classical secretion pathway for the proper dissemination of newly translated proteins throughout the cell and it has been thought that this pathway was the route to the cell surface (McConville et al., 2002). However, of the seven reports on Leishmania secreted proteins, only one found that a large proportion of the secreted proteins identified contained an $\mathrm{N}$-terminal secretion signal peptide for classical secretion (Paape et al., 2010). In all of the remaining reports, the authors observed the opposite to be true, with a majority of secretome proteins lacking a secretion signal peptide (Revest et al., 2008; Silverman et al., 2008, 2010a; DebRoy et al., 2010; Kima et al., 2010; Hassani et al., 2011). Three of the seven reports which investigated alternative mechanisms of secretion identified vesicles secreted by Leishmania (Silverman et al., 2008, 2010a; Hassani et al., 2011). Moreover, another report that investigated the intracellular localization of secreted proteins found the majority of the proteins examined associated with vesicular structures in the parasite as well as in infected host cells (Kima et al., 2010). Though in the nascent stages of discovery, the role of Leishmania secreted vesicles in pathogenesis has been investigated both directly and indirectly (Gomez et al., 2009; Halle et al., 2009; Silverman et al., 2010a,b; Hassani et al., 2011). In this review we 
will discuss what is currently known about the role that secreted vesicles play in Leishmania pathogenesis.

\section{MICROVESICLE RELEASE BY LEISHMANIA}

In eukaryotic cells secreted vesicles are formed either at the plasma membrane, referred to as "PM blebs," or in the lumen of various subcellular organelles - including endosomes, the trans-Golgi, and lysosomes-which are generally termed "exosomes" upon release from the cell. Due to the mechanism of their biogenesis, both types of vesicles (PM blebs and exosomes) contain cytosol and display the extracellular plasma membrane leaflet (which is also the luminal leaflet of the origin intracellular vesicles) on their outer surface. As a result the vesicles are oriented in the same manner as the cella characteristic that may well be central to their function(s) during infection.

Exosomes are formed by invagination of the limiting membrane of intracellular organelles, resulting in the formation of multi-vesicular bodies (MVB; Figure 1). Exosomes are released into the extracellular space upon fusion of the MVB with the plasma membrane. These vesicles are $50-100 \mathrm{~nm}$ in diameter and are of a specific density (Keller et al., 2006). One of the seven reports that investigated protein secretion by L. donovani concluded that these organisms were releasing bona fide exosomes (Silverman et al., 2010a). This conclusion was based primarily on comparison of the vesicles collected from Leishmania conditioned medium to mammalian exosomes. Leishmania vesicles were found to be nearly identical in morphology and density to mammalian exosomes (Figure 1) and the protein content of the Leishmania secreted vesicles was found to overlap with the canonical mammalian exosome proteome (Simpson et al., 2008) by more that 50\% (Silverman et al., 2008, 2010a). Likewise, homologs of a subset of the proteins known to regulate exosome biogenesis and release were identified in the Leishmania exosomes (Silverman et al., 2010a).

Another report on Leishmania protein secretion showed the plasma membrane of L. mexicana promastigotes to be decorated with vesicles, the number of which increased when the parasites were subjected to a short $37^{\circ} \mathrm{C}$ heat shock (Hassani et al., 2011). These vesicles were also collected from the Leishmania conditioned medium and imaged (Figure 1). This showed that they
A
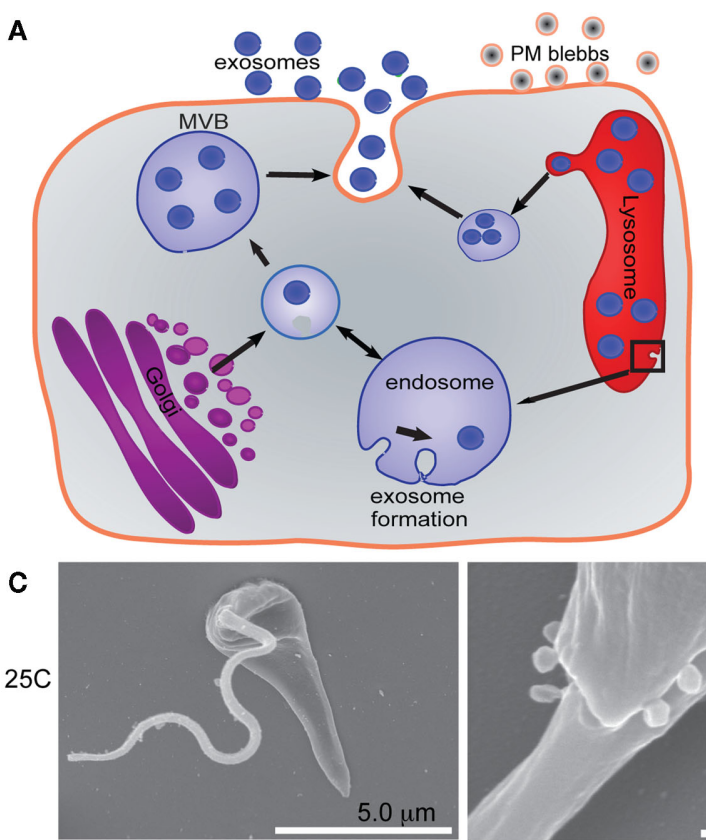

D

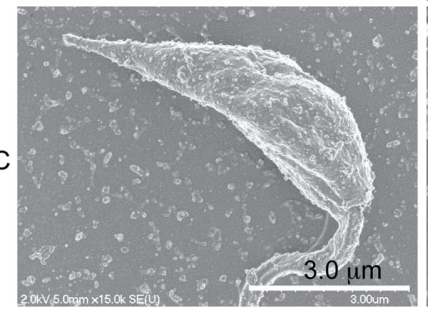

B

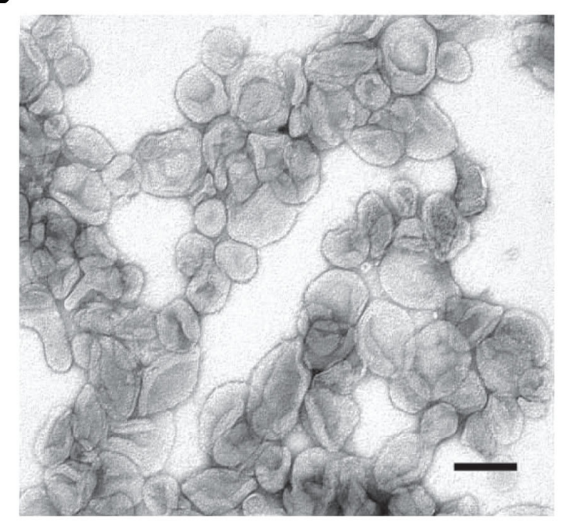

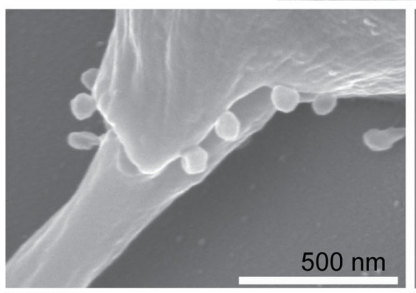
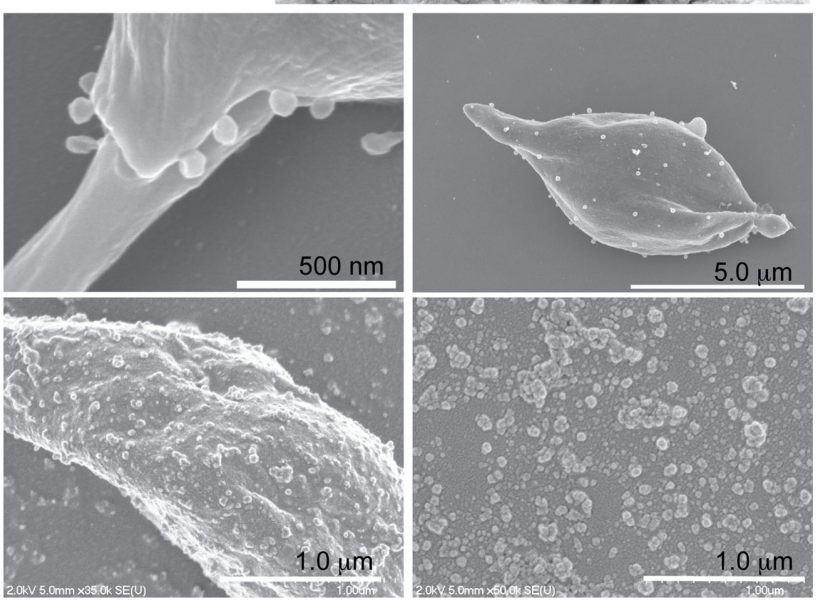

FIGURE 1 | Leishmania secrete microvesicles that are likely a combination of exosomes and plasma membrane blebs. (A) Cartoon illustrating the exosome secretion pathway and plasma membrane blebbing. (B) Leishmania exosomes were isolated and purified in a sucrose gradient prior to imaging by TEM. Scale bar $=100 \mathrm{~nm}$. Reprinted with permission from (Silverman et al., 2010a). (C) Scanning electron micrographs of Leishmania promastigotes (left and middle panel) and axenic amastigotes (far right panel) shows vesicles at the flagellar pocket and decorating the cell membrane. Reprinted with permission from (Silverman et al., 2008). (D) Scanning electron micrographs of Leishmania promastigotes (left and center panels) after a $4 \mathrm{~h}$ $37^{\circ} \mathrm{C}$ heat shock and the vesicles collected from the media during that time (right hand panel). Reprinted with permission from Hassani et al. (2011). 
had a similar morphology to the previously described Leishmania exosomes (Silverman et al., 2010a) with the exception that they had a markedly larger size range, with some of the vesicles almost $0.2 \mu \mathrm{m}$ in diameter - fourfold larger than the average exosome (Bobrie et al., 2011). Scanning electron micrographs of L. donovani axenic amastigotes after 1 week of axenic culture show similar vesicles decorating the cell surface (Silverman et al., 2008). It is possible that in both of these cases the vesicles were not exosomes per se, but possibly PM blebs. PM blebs are formed by budding or shedding from the plasma membrane and are known to be heterogeneous in size and density and to be released under cellular stress (Mathivanan et al., 2010).

No publication to date has unambiguously determined whether Leishmania secreted vesicles are of intracellular or plasma membrane origin. We propose that Leishmania are capable of secreting both types of vesicles, as mammalian cells are, and that each plays a role in pathogenesis. In fact, given that both PM blebs and exosomes contain cytosol and display the outer leaflet of the plasma membrane on their surface, it is possible that the vesicles formed intracellularly and those formed at the plasma membrane play overlapping roles when interacting with the host.

\section{THE PROTEIN CARGO AND SECRETION OF MICROVESICLES BY LEISHMANIA IS INFLUENCED BY THE HOST ENVIRONMENT}

If Leishmania secreted vesicles are involved in pathogenesis, one would expect that the mammalian host environment, which is at a higher temperature and lower $\mathrm{pH}$ than the gut of the sandfly vector, would affect vesicle release, vesicle cargo or both. Two independent reports now claim to have observed temperaturedependent vesicle release by Leishmania (Silverman et al., 2010a; Hassani et al., 2011). It has been shown that vesicle release by $L$. donovani increased threefold when stationary phase promastigotes were exposed to heat shock mimicking human body temperature, $37^{\circ} \mathrm{C}$, for $24 \mathrm{~h}$ (Silverman et al., 2010a). A more recent report similarly found significant temperature induced secretion of vesicles after only $4 \mathrm{~h}$ of heat shock (Hassani et al., 2011). Furthermore, quantitative proteomic analysis found the protein cargo of Leishmania exosomes to be both temperature and $\mathrm{pH}$ sensitive (Silverman et al., 2010a). For example, functional characterization of Leishmania exosomes released under infection-like conditions $\left(37^{\circ} \mathrm{C}\right.$ and acidic $\left.\mathrm{pH}\right)$ showed that exosomes harvested at $37^{\circ} \mathrm{C}$ at neutral $\mathrm{pH}$ were enriched in Kinase Activity related proteins and in direct contrast vesicles harvested in acidic $\mathrm{pH}$ were enriched in Phosphatase Activity (Silverman et al., 2010a). Likewise, analysis of the brief $(4 \mathrm{~h})$ heat shock induced "exoproteome" found a large portion of those proteins to have Catalytic Activity (Hassani et al., 2011). This specific packaging of individual proteins and functional groups, may likely reflect a sophisticated packaging of virulence factors by Leishmania in response to specific environments. Table 1 lists a small selection of the most interesting candidate virulence factors identified in Leishmania exosomes based on their increased secretion at $37^{\circ} \mathrm{C}$ or at acidic $\mathrm{pH}$. Additional study is required to elucidate the role of individual exosomal proteins in Leishmania pathogenesis. In any case, the temperature-dependent increase in vesicle release - along with the environment-dependent differences in protein content - support the conclusion that Leishmania exosome-mediated protein secretion is an active process subject to regulation by environmental signals.

\section{LEISHMANIA SECRETED VESICLES DELIVER CARGO TO HOST CELLS}

The specific up-regulation in vesicle release induced by infectionlike temperatures suggests that the parasites release vesicles into the extracellular milieu prior to invasion of a host cell [macrophage, neutrophil, or dendritic cell (DC)].These vesicles could be released from either recently inoculated promastigotes (and might actually be part of the inoculum, after secretion by metacyclic Leishmania in the sandfly salivary gland), by free amastigotes after cell rupture, or both. Following release, these exosomes would be free to interact with naïve host cells, either through surface binding, fusion with the plasma membrane, or by endocytosis. As illustrated in Figure 2, internalization of Leishmania exosomes from the extracellular environment by naïve cells has been directly observed (Silverman et al., 2010a). Furthermore, secretion of vesicular structures by Leishmania into the cytoplasmic compartment of an infected host has also been demonstrated (Silverman et al., 2010a).

We have observed exosomal delivery of cargo including GP63 and heat shock protein 10 (HSP10) to macrophages (Silverman et al., unpublished data). Additionally indirect evidence supporting

Table 1 | Leishmania secreted vesicles carry candidate virulence factors.

\begin{tabular}{|c|c|c|c|c|}
\hline Functional class & GeneDB Accession No. & Protein identity & Enriched in acidic pH & Reference \\
\hline \multirow[t]{3}{*}{$\begin{array}{l}\text { Immune evasion/ } \\
\text { suppression }\end{array}$} & LmjF10.0460 & GP63, leishmanolysin & & $\begin{array}{l}\text { Joshi et al. (2002), Gomez et al. } \\
\text { (2009), Halle et al. (2009) }\end{array}$ \\
\hline & LmjF26.0620 & Heat shock protein 10 & + & $\begin{array}{l}\text { Galli et al. (1996); Johnson et al. } \\
\text { (2005) }\end{array}$ \\
\hline & LmjF26.1240 & Heat shock protein 70 & & Elsner et al. (2007) \\
\hline \multirow{2}{*}{$\begin{array}{l}\text { Intracellular } \\
\text { survival }\end{array}$} & LmjF15.1040 & TRYP1, tryparedoxin peroxidase & + & Reiner and Malemud (1985) \\
\hline & LmjF11.0350 & 14-3-3 like protein & + & Porter et al. (2006) \\
\hline \multirow[t]{3}{*}{ T cell antigens } & LmjF35.2210 & Kinetoplastid membrane protein-11 & & Carvalho et al. (2005) \\
\hline & LmjF28.2740 & Activated protein kinase c receptor (LACK) & & Carvalho et al. (2005) \\
\hline & LmjF08.1110 & Stress-induced protein sti1 & & Webb et al. (1997) \\
\hline
\end{tabular}



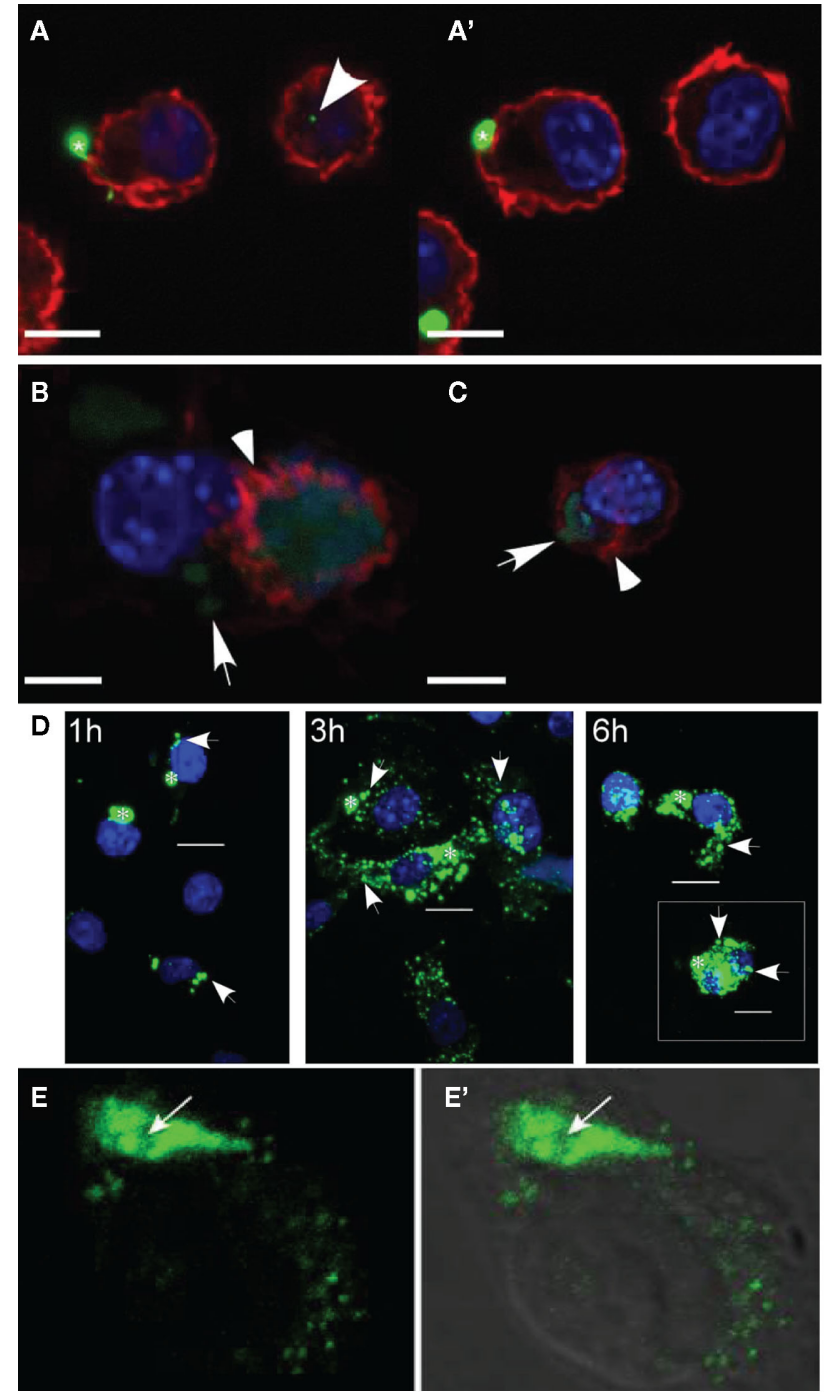

FIGURE 2 | Leishmania exosomes are engulfed by macrophages and their cargo access host cell cytosol. $\left(\mathbf{A}, \mathbf{A}^{\prime}\right)$ Sequential $z$-plane images of macrophages infected with Leishmania expressing GFP. Concave arrowhead indicates punctate Leishmania fluorescence that has been taken up by an uninfected macrophage. (B,C) Single $z$-place images of macrophages treated with purified Leishmania exosomes (arrows) expressing GFP. Red: host actin (arrowheads), blue: nuclei. Scale bars: $\left.\left(\mathbf{A}, \mathbf{A}^{\prime}\right)=9 \mu \mathrm{m}, \mathbf{B}\right)=5.4 \mu \mathrm{m}$,

(C) $=13 \mu \mathrm{m}$. (A, $\left.\mathbf{A}^{\prime}, \mathbf{B}, \mathbf{C}\right)$ are all reprinted with permission from Silverman et al. (2010a). (D) Promastigotes with FITC-labeled surface proteins were added to macrophages and infected cells were processed for confocal microscopy at 1 , 3 , and $6 \mathrm{~h}$ post infection $6 \mathrm{~h}$ insert is another representative cell from the

the ability of exosomes to deliver effector cargo to naïve cells has been published. Macrophages infected with L. major have parasitederived GP63, a Leishmania virulence factor, in their cytosolic compartment and also associated with the inner leaflet of the plasma membrane plasma membrane (Figure 2; Gomez et al., 2009). Notably, GP63 was similarly found in these same subcellular locales when naïve macrophages were incubated with Leishmania cell-free culture supernatant (Figure 2; Gomez et al., 2009). Based on our published results, as well as those of Hassani et al. the
$\mathbf{F}$
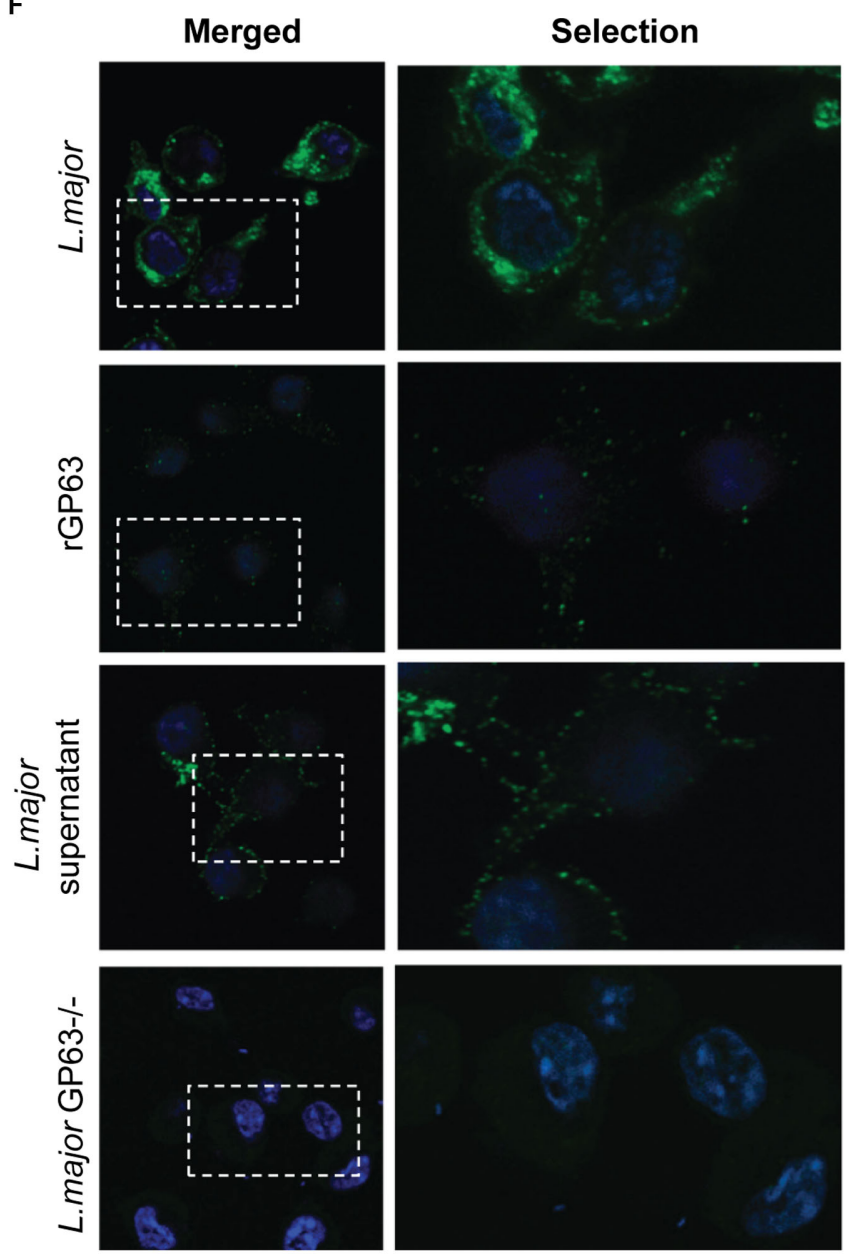

same slide). White arrowheads indicate exported proteins. Scale bars $=50 \mu \mathrm{m}$. (E,E') Macrophages infected with $L$. donovani were processed for confocal microscopy and probed with and antibody specific to Leishmania $E F-1 \alpha$. $\left(E^{\prime}\right)$ is the merge of the fluorescence image with a differential interference contrast images. Arrows mark the localization of Leishmania in the infected cell. Reprinted with permission from Nandan et al. (2002).

(F) Macrophages were incubated with $1 \mathrm{mg}$ of rGP63 or were infected with $L$. major, or L. major GP63 ${ }^{-/-}$, or treated with L. major cell-free culture supernatant for $1 \mathrm{~h}$. Intracellular GP63 was evaluated by confocal microscopy using anti-GP63 antibodies (green). Leishmania and macrophage nuclei are stained blue. Reprinted with permission from Gomez et al. (2009).

culture supernatants used by Gomez et al. clearly must have contained secreted vesicles carrying GP63 (Silverman et al., 2010a; Hassani et al., 2011). As such the cytosolic location of the GP63 after incubation with culture supernatant is very likely to be the result of exosome-mediated delivery of the protein. Likewise, in the immunofluorescence experiments carried out the GP63 fluorescence was punctate, suggesting that host cells engulfed GP63 in a vesicular fashion (Gomez et al., 2009). This punctate fluorescence is very similar to that which was observed in the characterization 
of Leishmania exosome uptake by macrophages (Silverman et al., 2010a). Furthermore, it was found that treatment with the cholesterol chelator methyl- $\beta$-cyclodextrin known to disrupt lipid rafts and exosome release and uptake (Keller et al., 2006) - blocked macrophage uptake of GP63 (Gomez et al., 2009). Taken together, these findings show that GP63 is (1) secreted by Leishmania in vesicles, (2) delivered to host cytosol, (3) taken up in a vesicular manner, and (4) that uptake is blocked by reagents which affect vesicle uptake. The data suggest a model in which exosomes are involved in parasite-to-host delivery of GP63.

GP63 is not the only protein cargo of Leishmania exosomes to have been found in the cytosol of host cells. Elongation factor- $1 \alpha$ (EF-1 $\alpha$; Figure 2) and the heat shock proteins 90 and 70 have also been detected in the cytosol of Leishmania-infected macrophages (Nandan et al., 2002; Silverman et al., 2010a), suggesting that perhaps these vesicles are also involved in delivery of effectors to host cytosol after infection has taken hold.

\section{LEISHMANIA SECRETED VESICLES HAVE IMMUNOMODULATORY PROPERTIES}

We have proposed a model suggesting that early during infection Leishmania secreted vesicles interact with and functionally prime naïve host cells in the local environment for infection. In support of this model, it is now known that Leishmania secreted vesicle treatment brings about clear changes in host cell phenotype. L. donovani exosomes induced production of the neutrophil chemokine IL- 8 by differentiated THP-1 macrophages (Silverman et al., 2010a). A model has recently emerged suggesting that Leishmania use neutrophils as Trojan horses to deliver themselves to macrophages via a "silent phagocytosis" pathway, thus avoiding cell activation (van Zandbergen et al., 2004). Exosome induced IL-8 secretion may likely bring about the early recruitment of neutrophils to the site of infection (Peters et al., 2008; Peters and
Sacks, 2009) and in this manner contribute to development of early infection. It is worth noting that incubation of primary human monocytes with Leishmania exosomes did not induce IL-8 production (Silverman et al., 2010b) and this discrepancy may be related to specific stages of monocyte/macrophage differentiation. In any case, under appropriate local conditions the induction of IL- 8 release by exosomes may promote pathogenesis.

Beyond IL-8, the cytokine IFN- $\gamma$ is widely considered to be crucial for activating macrophages and clearing leishmanial infections (Nandan and Reiner, 1995). To test the hypothesis that exosomes prime host cells for infection, we treated primary human monocytes with exosomes to infection with virulent Leishmania and then subsequently treated those cells with IFN- $\gamma$ (Silverman et al., $2010 \mathrm{~b})$. The results showed that cells that were infected after exosome treatment had blunted responses to IFN- $\gamma$ for the induction of the proinflammatory cytokines TNF- $\alpha$ and IL-8 (Figure 3). In contrast, exposure of monocytes to exosomes prior to infection significantly potentiated IFN- $\gamma$-induced IL-10 production (Silverman et al., 2010a). Given that IL-10 is a potent anti-inflammatory cytokine involved in mediating immune suppression during Leishmania infection (Murray et al., 2002; Anderson et al., 2008, 2009), enhanced production of IL-10 as a result of exposure to exosomes suggests a significant role for these vesicles in pathogenesis.

Gomez et al. (2009) published a study focused on cell signaling which provides additional support for a model in which Leishmania exosomes attenuate IFN- $\gamma$-induced macrophage cytokine expression. The opposing functions of protein-tyrosine kinases (PTKs) - activators - and protein-tyrosine phosphatases (PTPs) deactivators control much of a cell's biological processes. It has been known for some time that tyrosine phosphorylation in Leishmania infected macrophages is generally impaired as a result of Leishmania activation of the Src homology-2 domain-containing phosphatase-1 (SHP-1; Blanchette et al., 1999; Nandan et al., 1999,

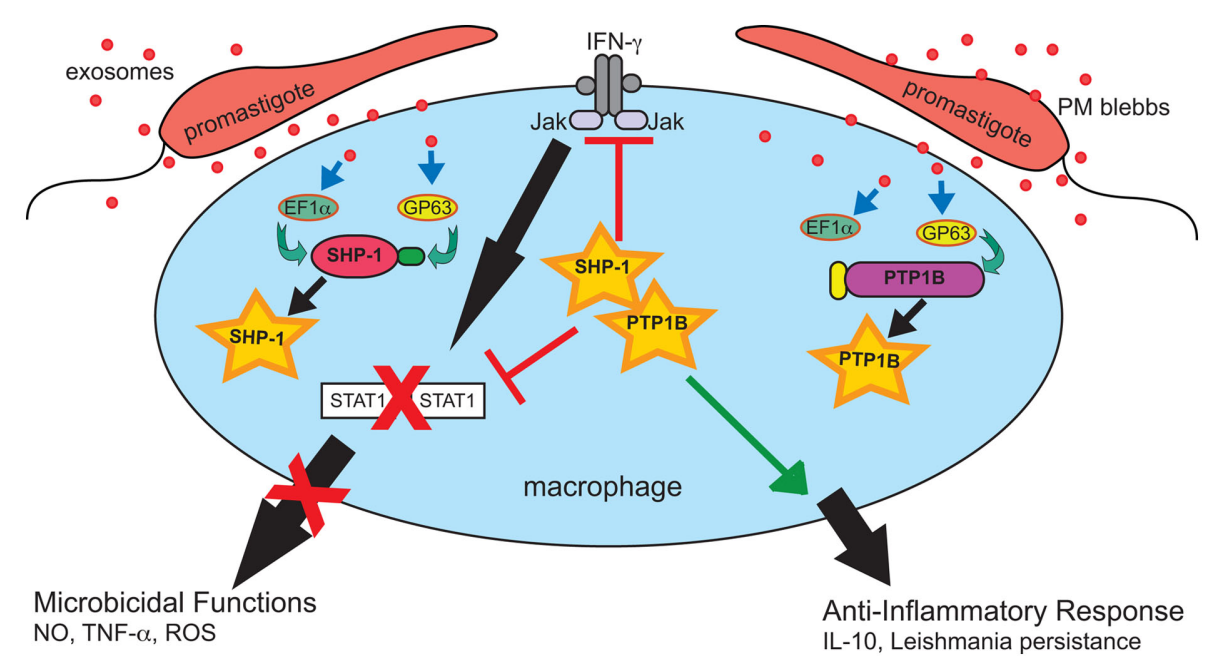

FIGURE 3 | Leishmania exosomes deliver preemptive strikes targeting host cell signaling machinery to create an anti-inflammatory pro-parasitic environment for eventual Leishmania invasion and the establishment of infection. Exosomes deliver effector compounds, such as GP63 and EF-1 $\alpha$, to target host cells, including macrophages. This results in the activation of multiple protein-tyrosine phosphatases, e.g., SHP-1 and PTP1B, which dephosphorylate key targets in critical signaling pathways including the IFN- $\gamma /$ Jak-STAT1 pathway. This disrupts signal transduction and leads to inhibition of macrophage microbicidal functions. Reprinted with permission and modifications from Silverman and Reiner (2011). 
2002).Gomez et al. (2009) found that, similar to Leishmania infection, cell-free culture supernatant from L. mexicana and L. major was capable of cleaving and activating various host macrophage PTPs including SHP-1, PTP1B, and T cell PTP. Notably, activation occurred almost immediately; cleavage products were detected after only $1 \mathrm{~min}$ of exposure to Leishmania culture supernatant and were enzymatically active (Gomez et al., 2009). It can be assumed based on subsequent work from this same laboratory, as well as our own work, that this culture supernatant contained secreted vesicles (Silverman et al., 2010a; Hassani et al., 2011). Leishmania GP63, cargo of Leishmania exosomes, was found to be required and responsible for the cleavage and activation of PTPs (Gomez et al., 2009). This activity, in addition to GP63 cytosolic delivery, was blocked by treatment with methyl- $\beta$-cyclodextrin (Gomez et al., 2009), which is known to disrupt both lipid rafts and exosome trafficking (Lancaster and Febbraio, 2005; Keller et al., 2006). Macrophages infected with Leishmania have been shown to exhibit reduced phosphorylation of Jak tyrosine kinases and deficient production of nitric oxide (NO) in response to IFN$\gamma$ treatment and both of these abnormalities are believed to be brought about as downstream effects of PTP activation (Gregory and Olivier, 2005; Nandan and Reiner, 2005). As illustrated in Figure 3, the functional result of this Leishmania-induced PTP activity is an impaired IFN- $\gamma$ signaling cascade required for proper macrophage activation (Nandan and Reiner, 1995; Blanchette et al., 1999; Nandan et al., 1999, 2002; Forget et al., 2005). Gomez et al. (2009) showed these phenotypic changes to be dependent on GP63 as well as host SHP-1 and PTP1B. Taken together, these findings demonstrate that cytosolic delivery of GP63, most likely as exosome cargo, is required to bring about activation of host cell PTPs to bring about negative regulation of macrophage activation in response to IFN- $\gamma$.

Interestingly, GP63 is not the only Leishmania protein secreted in exosomes known to activate PTPs. Nandan et al. found that Leishmania EF-1 $\alpha$ specifically activated SHP-1 in vitro and in vivo. In addition, this group found that delivery of Leishmania EF-1 $\alpha$ to macrophages using the protein delivery system Profect (which is incidentally based on lipid vesicles - similar to exosomes) prevented the induction of iNOS in response to treatment with IFN- $\gamma$ (Nandan et al., 2002). Nandan et al. also found that Leishmania EF-1 $\alpha$ translocated from the phagolysosome into the cytoplasmic compartment of infected cells with a punctuate vesicular morphology (Figure 2) and more recent work showed EF-1 $\alpha$ to be a common component of Leishmania exosomes (Nandan et al., 2002; Silverman et al., 2010a).

Another interesting finding that has emerged from this work, is that HSP $100^{-/-}$L. donovani produced vesicles apparently lacking GP63 and EF-1 $\alpha$. Though similar to wild-type (WT) exosomes in terms of morphology and biochemistry, HSP100 knock-out exosomes were stimulatory for cytokine production both in monocyte-derived dendritic cells (MoDCs) and monocytes (Silverman et al., 2010b).This may reflect the inability of these exosomes to activate PTPs with their inhibitory effect due to the absence of GP63 (Gomez et al., 2009; Halle et al., 2009). Moreover, when MoDCs were exosome treated and then co-cultured with naïve CD4+ T lymphocytes, the number of IFN- $\gamma$ producing $\mathrm{T}$ cells that emerged was two times greater when the
MoDCs were treated with $\mathrm{HSP} 100^{-1-}$ vesicles as compared to treatment with WT exosomes (Silverman et al., 2010b). Notably, the enhanced stimulatory capacity of $\mathrm{HSP}_{100^{-1-}}$ exosomes did not involve changes in the expression of co-stimulatory markers (CD80, CD86, and HLA-DR) by exosome treated MoDCs when compared with WT exosomes (Silverman et al., 2010b). Presently, the mechanistic basis for these results are not understood although it is highly likely that differences in protein cargo of the vesicles are involved - and likely cargo dependent effects including those of GP63's on PTPs activities.

In contrast to the findings with $\mathrm{HSP} 100^{-/-}$exosomes, vesicles harvested from lipophosphoglycan (LPG) 2 null Leishmania behaved liked their WT counterparts in all of the above experiments (Silverman et al., 2010b). Presumably the LPG2-1- vesicles are lacking in LPG only and contain GP63 and EF-1 $\alpha$ (Spath et al., 2003; Silverman et al., 2010b), which may account for their WTlike properties, although this was not explicitly examined. These findings suggest that the null or inhibitory phenotypes of WT and LPG $2^{-1-}$ exosomes are reflections of the specific protein composition of these vesicles - and potentially reliant on GP63, EF- $1 \alpha$, or both. Furthermore, the delivery of inhibitory cargo by Leishmania exosomes including both packaging and secretion appears to be dependent upon HSP100.

\section{LEISHMANIA EXOSOMES PRODUCE PERMISSIVE ENVIRONMENTS FOR PARASITE INFECTION IN VIVO}

The results discussed above support a model in which Leishmania exosomes deliver virulence factors - GP63, EF-1 $\alpha$, and others - to host cells and interfere with host cell signaling pathways required to control infection. In vivo investigations of the immunomodulatory properties of Leishmania exosomes, using mouse models of infection provide further evidence to support this model (Silverman et al., 2010b). Exposure of resistant C57BL/6 mice to WT $L$. donovani exosomes resulted in higher parasite loads in the spleen after subsequent infection and a cytokine profile suggestive of an immune response dominated by suppressor T cells producing both IL-10 and IFN- $\gamma$ (Silverman et al., 2010b). Th1 cells dually producing IL-10 and IFN- $\gamma$ have recently been shown to be the dominant suppressor cell in Leishmania infections (Anderson et al., 2007; Nylen et al., 2007), and high IFN- $\gamma$ production has recently been linked to generation of suppressor cell types (Eljaafari et al., 2009). Moreover, the pro-parasitic properties of WT exosomes have been shown to cross Leishmania species. Thus, similar to the results for $L$. donovani exosomes, exposure of susceptible BALB/c mice to $L$. major exosomes prior to infection exacerbated disease progression, and this appeared to involve promotion of Th2 polarization (Silverman et al., 2010b). In both of these experimental models, the mice were infected for 4 weeks prior to examining parasite loads and hence provide a window on exosome effects during early infection. Longer term infection studies will be required to examine the in vivo effects of exosomes on chronic infection. Nevertheless, these in vivo findings indicate that Leishmania exosomes are capable of biasing the immune response to make it permissive for infection, perhaps through their generally inhibitory effects on monocytes as well as MoDCs.

In vivo data with PTP knock-out mice further supports this conclusion. L. major infection of either PTP1B null mice or SHP-1 
null mice resulted in delayed footpad thickening and decreased parasite burden - though only during the early stages of infection, as well as greater leukocyte recruitment (Forget et al., 2001; Gomez et al., 2009). This demonstrates a clear role for these PTPs in creating a parasite permissive environment early during infection. Furthermore, these effects were shown to be GP63 dependent (Gomez et al., 2009). These in vivo findings suggest that GP63dependent cleavage and activation of host PTPs plays a crucial role during the early, stages of the disease, perhaps reflecting the exosome-mediated delivery of GP63 and early priming of target cells for infection.

\section{CONCLUSION}

The findings reviewed above support a model in which exosomemediated delivery of GP63 and EF- $1 \alpha$ to target cell cytosol activates host PTPs, which prevent IFN- $\gamma$-induced signaling and blunt the pro-inflammatory cytokine response to infection. The

\section{REFERENCES}

Anderson, C. F., Lira, R., Kamhawi, S., Belkaid, Y., Wynn, T. A., and Sacks, D. (2008). IL-10 and TGFbeta control the establishment of persistent and transmissible infections produced by Leishmania tropica in C57BL/6 mice. J. Immunol. 180, 4090-4097.

Anderson, C. F., Oukka, M., Kuchroo, V. J., and Sacks, D. (2007). CD4(+)CD25(-)Foxp3(-) Th1 cells are the source of IL-10mediated immune suppression in chronic cutaneous leishmaniasis. J. Exp. Med. 204, 285-297.

Anderson, C. F., Stumhofer, J. S., Hunter, C. A., and Sacks, D. (2009). IL27 regulates IL-10 and IL-17 from CD4+ cells in nonhealing Leishmania major infection. J. Immunol. 183, 4619-4627.

Besteiro, S., Williams, R. A., Morrison, L. S., Coombs, G. H., and Mottram, J. C. (2006). Endosome sorting and autophagy are essential for differentiation and virulence of Leishmania major. J. Biol. Chem. 281, 11384-11396.

Blanchette, J., Racette, N., Faure, R., Siminovitch, K. A., and Olivier, M. (1999). Leishmaniainduced increases in activation of macrophage SHP-1 tyrosine phosphatase are associated with impaired IFN-gamma-triggered JAK2 activation. Eur. J. Immunol. 29, 3737-3744.

Bobrie, A., Colombo, M., Raposo, G., and Théry, C. (2011). Exosome secretion: molecular mechanisms and roles in immune responses. Traffic 12, 1659-1668.

Carvalho, L. P., Passos, S., Dutra, W. O., Soto, M., Alonso, C., Gollob, K. J., Carvalho, E. M., and
Ribeiro De Jesus, A. (2005). Effect of LACK and KMP11 on IFNgamma production by peripheral blood mononuclear cells from cutaneous and mucosal leishmaniasis patients. Scand. J. Immunol. 61, 337-342.

DebRoy, S., Keenan, A. B., Ueno, N., Jeronimo, S. M. B., Donelson, J. E., and Wilson, M. E. (2010). Leishmania infantum chagasi: a genomebased approach to identification of excreted/secreted proteins. Exp. Parasitol. 126, 582-591.

Eljaafari, A., Li, Y. P., and Miossec, P. (2009). IFN-gamma, as secreted during an alloresponse, induces differentiation of monocytes into tolerogenic dendritic cells, resulting in FoxP3+ regulatory $\mathrm{T}$ cell promotion. J. Immunol. 183, 2932-2945.

Elsner, L., Muppala, V., Gehrmann, M., Lozano, J., Malzahn, D., Bickeboller, H., Brunner, E., Zientkowska, M., Herrmann, T., Walter, L., Alves, F., Multhoff, G., and Dressel, R. (2007). The heat shock protein HSP70 promotes mouse NK cell activity against tumors that express inducible NKG2D ligands. J. Immunol. 179, 5523-5533.

Forget, G., Matte, C., Siminovitch, K. A., Rivest, S., Pouliot, P., and Olivier, M. (2005). Regulation of the Leishmania-induced innate inflammatory response by the protein tyrosine phosphatase SHP-1. Eur. J. Immunol. 35, 1906-1917.

Forget, G., Siminovitch, K. A., Brochu, S., Rivest, S., Radzioch, D., and Olivier, M. (2001). Role of host phosphotyrosine phosphatase SHP1 in the development of murine leishmaniasis. Eur. J. Immunol. 31, 3185-3196.

data strongly suggest that Leishmania secreted vesicles are capable of delivering effector molecules to naïve host cells which in turn modulate the immune response in a pro-parasitic manner. Leishmania secreted vesicles and their effector cargo are likely essential events to establish early stage infection and set the stage for subsequent events leading to the development of chronic infection.

\section{ACKNOWLEDGMENTS}

The authors would like to acknowledge the following individuals for their contributions to studies cited in this review: Ulrike Lambertz, Emily Thi, Devki Nandan, Joachim Clos, Leonard Foster, and Megan Levings. In addition, we would like to thank Martin Olivier for generously allowing us to reprint important figures. Neil Reiner is supported by the Canadian Institutes of Health Research grant MOP-84582 and Judith Silverman is supported by a grant from PrioNet Canada.

Galli, G., Ghezzi, P., Mascagni, P., Marcucci, F., and Fratelli, M. (1996). Mycobacterium tuberculosis heat shock protein 10 increases both proliferation and death in mouse P19 teratocarcinoma cells. In vitro Cell. Dev. Biol. Anim. 32, 446-450.

Gomez, M. A., Contreras, I., Halle, M., Tremblay, M. L., Mcmaster, R. W., and Olivier, M. (2009). Leishmania GP63 alters host signaling through cleavage-activated protein tyrosine phosphatases. Sci. Signal. 2, ra58.

Gregory, D. J., and Olivier, M. (2005). Subversion of host cell signalling by the protozoan parasite Leishmania. Parasitology 130(Suppl.), S27-S35.

Halle, M., Gomez, M. A., Stuible, M., Shimizu, H., Mcmaster, W. R., Olivier, M., and Tremblay, M. L. (2009). The Leishmania surface protease GP63 cleaves multiple intracellular proteins and actively participates in p38 mitogen-activated protein kinase inactivation. J. Biol. Chem. 284, 6893-6908.

Hassani, K., Antoniak, E., Jardim, A., and Olivier, M. (2011). Temperature-induced protein secretion by Leishmania mexicana modulates macrophage signalling and function. PLoS ONE 6, e18724. doi:10.1371/journal.pone.0018724

Hubel, A., Krobitsch, S., Horauf, A., and Clos, J. (1997). Leishmania major Hsp100 is required chiefly in the mammalian stage of the parasite. Mol. Cell. Biol. 17, 5987-5995.

Jaramillo, M., Gomez, M. A., Larsson, O., Shio, M. T., Topisirovic, I., Contreras, I., Luxenburg, R., Rosenfeld, A., Colina, R., Mcmaster, R. W., Olivier, M., Costa-Mattioli, M. and Sonenberg, N. (2011). Leishmania repression of host translation through mTOR cleavage is required for parasite survival and infection. Cell Host Microbe 9, 331-341.

Johnson, B. J., Le, T. T., Dobbin, C. A., Banovic, T., Howard, C. B., Flores Fde, M., Vanags, D., Naylor, D. J., Hill, G. R., and Suhrbier, A. (2005). Heat shock protein 10 inhibits lipopolysaccharide-induced inflammatory mediator production. J. Biol. Chem. 280, 4037-4047.

Joshi, P. B., Kelly, B. L., Kamhawi, S., Sacks, D. L., and Mcmaster, W. R. (2002). Targeted gene deletion in Leishmania major identifies leishmanolysin (GP63) as a virulence factor. Mol. Biochem. Parasitol. 120, 33-40.

Keller, S., Sanderson, M. P., Stoeck, A., and Altevogt, P. (2006). Exosomes: from biogenesis and secretion to biological function. Immunol. Lett. 107, 102-108.

Kima, P. E., Bonilla, J. A., Cho, E. Ndjamen, B., Canton, J., Leal, N., and Handfield, M. (2010). Identification of Leishmania proteins preferentially released in infected cells using change mediated antigen technology (CMAT). PLoS Negl. Trop. Dis. 4, e842. doi:10.1371/journal.pntd.0000842

Klein, C., Gopfert, U., Goehring, N., Stierhof, Y. D., and Ilg, T. (1999). Proteophosphoglycans of Leishmania mexicana. Identification, purification, structural and ultrastructural characterization of the secreted promastigote proteophosphoglycan pPPG2, a stage-specific glycoisoform of amastigote aPPG. Biochem. J. 344(Pt 3), 775-786.

Lancaster, G. I., and Febbraio, M. A. (2005). Exosome-dependent trafficking of HSP70: a novel secretory pathway for cellular stress proteins. J. Biol. Chem. 280, 23349-23355. 
Lieke, T., Nylen, S., Eidsmo, L., Mcmaster, W. R., Mohammadi, A. M., Khamesipour, A., Berg, L., and Akuffo, H. (2008). Leishmania surface protein gp63 binds directly to human natural killer cells and inhibits proliferation. Clin. Exp. Immunol. 153, 221-230.

Mathivanan, S., Ji, H., and Simpson, R. J. (2010). Exosomes: extracellular organelles important in intercellular communication. J. Proteomics 73, 1907-1920.

McConville, M. J., Mullin, K. A., Ilgoutz, S. C., and Teasdale, R. D. (2002). Secretory pathway of trypanosomatid parasites. Microbiol. Mol. Biol. Rev. 66, 122-154.

Murray, H. W., Lu, C. M., Mauze, S., Freeman, S., Moreira, A. L., Kaplan, G., and Coffman, R. L. (2002). Interleukin-10 (IL-10) in experimental visceral leishmaniasis and IL-10 receptor blockade as immunotherapy. Infect. Immun. 70, 6284-6293.

Nandan, D., Lo, R., and Reiner, N. E. (1999). Activation of phosphotyrosine phosphatase activity attenuates mitogen-activated protein kinase signaling and inhibits c-FOS and nitric oxide synthase expression in macrophages infected with Leishmania donovani. Infect. Immun. 67, 4055-4063.

Nandan, D., and Reiner, N. E. (1995). Attenuation of gamma interferoninduced tyrosine phosphorylation in mononuclear phagocytes infected with Leishmania donovani: selective inhibition of signaling through Janus kinases and Stat1. Infect. Immun. 63, 4495-4500.

Nandan, D., and Reiner, N. E. (2005). Leishmania donovani engages in regulatory interference by targeting macrophage protein tyrosine phosphatase SHP-1. Clin. Immunol. 114, 266-277.

Nandan, D., Yi, T., Lopez, M., Lai, C., and Reiner, N. E. (2002). Leishmania EF-1alpha activates the Src homology 2 domain containing tyrosine phosphatase SHP-1 leading to macrophage deactivation. J. Biol. Chem. 277, 50190-50197.

Nylen, S., Maurya, R., Eidsmo, L., Manandhar, K. D., Sundar, S., and Sacks, D. (2007). Splenic accumulation of IL-10 mRNA in T cells distinct from CD4 +CD25 + (Foxp3) regulatory $\mathrm{T}$ cells in human visceral leishmaniasis. J. Exp. Med. 204, 805-817.

Paape, D., Barrios-Llerena, M. E., Le Bihan, T., Mackay, L., and Aebischer, T. (2010). Gel free analysis of the proteome of intracellular Leishmania mexicana. Mol. Biochem. Parasitol. 169, 108-114.

Peters, N. C., Egen, J. G., Secundino, N., Debrabant, A., Kimblin, N., Kamhawi, S., Lawyer, P., Fay, M. P., Germain, R. N., and Sacks, D. (2008). In vivo imaging reveals an essential role for neutrophils in leishmaniasis transmitted by sand flies. Science 321, 970-974.

Peters, N. C., and Sacks, D. L. (2009). The impact of vector-mediated neutrophil recruitment on cutaneous leishmaniasis. Cell. Microbiol. 11, 1290-1296.

Porter, G. W., Khuri, F. R., and Fu, H. (2006). Dynamic 14-3-3/client protein interactions integrate survival and apoptotic pathways. Semin. Cancer Biol. 16, 193-202.

Reiner, N. E., and Malemud, C. J. (1985). Arachidonic acid metabolism by murine peritoneal macrophages infected with Leishmania donovani: in vitro evidence for parasite-induced alterations in cyclooxygenase and lipoxygenase pathways. J. Immunol. 134, 556-563.

Revest, M., Donaghy, L., Cabillic, F., Guiguen, C., and Gangneux, J. P. (2008). Comparison of the immunomodulatory effects of $L$. donovani and L. major excretedsecreted antigens, particulate and soluble extracts and viable parasites on human dendritic cells. Vaccine 26 6119-6123.

Silverman, J. M., Chan, S. K., Robinson, D. P., Dwyer, D. M., Nandan, D., Foster, L. J., and Reiner, N. E. (2008). Proteomic analysis of the secretome of Leishmania donovani. Genome Biol. 9, R35.

Silverman, J. M., Clos, J., De'oliveira, C. C., Shirvani, O., Fang, Y., Wang, C., Foster, L. J., and Reiner, N. E. (2010a). An exosome-based secretion pathway is responsible for protein export from Leishmania and communication with macrophages. J. Cell. Sci. 123, 842-852.

Silverman, J. M., Clos, J., Horakova, E., Wang, A. Y., Wiesgigl, M., Kelly, I., Lynn, M. A., Mcmaster, W. R., Foster, L. J., Levings, M. K., and Reiner, N. E. (2010b). Leishmania exosomes modulate innate and adaptive immune responses through effects on monocytes and dendritic cells. J. Immunol. 5011-5022.

Silverman, J. M., and Reiner, N. E. (2011). Exosomes and other microvesicles in infection biology: organelles with unanticipated phenotypes. Cell. Microbiol. 13, 1-9.

Simpson, R. J., Jensen, S. S., and Lim, J. W. (2008). Proteomic profiling of exosomes: current perspectives. Proteomics 8, 4083-4099.

Spath, G. F., Garraway, L. A., Turco, S. J., and Beverley, S. M. (2003). The role(s) of lipophosphoglycan (LPG) in the establishment of Leishmania major infections in mammalian hosts. Proc. Natl. Acad. Sci. U.S.A. 100, 9536-9541.

van Zandbergen, G., Klinger, M., Mueller, A., Dannenberg, S., Gebert, A., Solbach, W., and Laskay, T. (2004). Cutting edge: neutrophil granulocyte serves as a vector for Leishmania entry into macrophages. J. Immunol. 173, 6521-6525.

Vannier-Santos, M. A., Martiny, A., Meyer-Fernandes, J. R., and De Souza, W. (1995). Leishmanial protein kinase $\mathrm{C}$ modulates host cell infection via secreted acid phosphatase. Eur. J. Cell Biol. 67, 112-119.

Webb, J. R., Campos-Neto, A., Skeiky, Y. A., and Reed, S. G. (1997). Molecular characterization of the heatinducible LmSTI1 protein of Leishmania major. Mol. Biochem. Parasitol. 89, 179-193.

Conflict of Interest Statement: The authors declare that the research was conducted in the absence of any commercial or financial relationships that could be construed as a potential conflict of interest.

Received: 23 September 2011; paper pending published: 11 November 2011; accepted: 23 December 2011; published online: 09 January 2012.

Citation: Silverman JM and Reiner NE (2012) Leishmania exosomes deliver preemptive strikes to create an environment permissive for early infection. Front. Cell. Inf. Microbio. 1:26. doi: 10.3389/fcimb.2011.00026

Copyright (C) 2012 Silverman and Reiner. This is an open-access article distributed under the terms of the Creative Commons Attribution Non Commercial License, which permits non-commercial use, distribution, and reproduction in other forums, provided the original authors and source are credited. 